\title{
Differences in cue-induced brain activation between long-term methadone maintenance treatment and protracted abstinence in heroin use disorder patients: a functional magnetic resonance imaging study
}

\author{
Xuan Wei ${ }^{1,2} \wedge$, Qiang $\mathrm{Li}^{1}$, Jiajie Chen ${ }^{1}$, Baorui Shen ${ }^{1,3}$, Wei Wang ${ }^{1}$, Wei Li $^{1}$ \\ ${ }^{1}$ Department of Radiology, Tangdu Hospital, Air Force Medical University, Xi'an, China; ${ }^{2}$ Department of Radiology, Beijing Friendship Hospital, \\ Capital Medical University, Beijing, China; ${ }^{3}$ Basic Medical College, Air Force Medical University, Xi'an, China
}

Correspondence to: Wei Li; Wei Wang. Department of Radiology, Tangdu Hospital, Air Force Medical University, 569 Xinsi Road, Baqiao District, Xi'an 710038, China. Email: tdliwei@126.com; tdwangw@126.com

\begin{abstract}
Background: Heroin use disorder (HUD) remains one of the gravest public health issues in China. Methadone maintenance treatment (MMT) and protracted abstinence (PA) are the most commonly used treatments for HUD. Although both treatment approaches can alleviate heroin cravings, a previous study found that MMT patients had stronger cue-induced brain activation than patients undergoing PA; however, the changes associated with long-term treatment are unclear.
\end{abstract}

Methods: Male patients with HUD who had been undergoing either PA ( $n=24)$ or MTT (n=21) for approximately 12 months, together with 20 demographically matched healthy controls, completed an eventrelated functional magnetic resonance imaging (fMRI) task. The subjective craving for heroin was evaluated using a visual analog scale.

Results: Compared to the healthy controls, the MMT and PA groups demonstrated significantly higher brain activation in the left pallidum, middle occipital gyrus, postcentral gyrus, anterior cingulate cortex, middle cingulate cortex, inferior parietal lobule, superior parietal lobule, amygdala, hippocampus, right inferior temporal gyrus, inferior frontal gyrus triangularis, and caudate during exposure to heroin-related cues. Compared to those undergoing PA, patients in the MMT group demonstrated significantly higher brain activation in all of these regions. Except for the left inferior parietal lobule and left superior parietal lobule, there were no statistically significant differences between the PA and healthy control groups. The MMT patients showed significantly higher subjective cravings before and after exposure to heroin cues than the PA group, but there was no significant difference in the change in subjective cravings between the 2 groups.

Conclusions: The results suggested that although the HUD patients receiving long-term MMT complied with the treatment, they still had higher subjective cravings and cue-induced brain activation than those undergoing PA. Therefore, long-term PA appears to be more beneficial than MMT in reducing the salience value of drug cues in patients with HUD.

Keywords: Heroin use disorder (HUD); protracted abstinence (PA); methadone maintenance treatment (MMT); craving; functional magnetic resonance imaging (fMRI)

Submitted Aug 25, 2020. Accepted for publication Dec 11, 2020.

doi: $10.21037 /$ qims-20-1002

View this article at: http://dx.doi.org/10.21037/qims-20-1002

\footnotetext{
^ ORCID: 0000-0002-5797-4928.
} 


\section{Introduction}

Drug addiction is defined as a "chronic, relapsing disorder" characterized by a compulsion to seek and use drugs (1). The current opioid epidemic is one of the most severe public health crises facing China. According to the latest Annual Report on Drug Control in China, methamphetamine has become the most abused drug in China. However, opioid abuse causes serious harm, and the number of patients with heroin use disorder (HUD) remains large $(2,3)$. Responding effectively to the opioid epidemic has proved difficult, because of its changing nature, its geographic and demographic diversity, the multiplicity of its causes, and the severity of adverse outcomes associated with opioid use and opioid use disorder (OUD) (4). The high relapse rate for compulsive drug use is a notable feature of drug addiction and is one of the biggest obstacles to abstinence (5-7).

Methadone maintenance treatment (MMT) and protracted abstinence (PA) are the 2 most commonly used treatments for HUD in China. Methadone acts as an opioid receptor agonist, and its effect of reducing cravings, physical withdrawal symptoms, and relapse behavior in individuals with HUD has been well documented $(8,9)$. MMT is reported to be one of the most effective treatments for HUD (10). PA is another major treatment method used in China; HUD patients are prohibited from using any illegal drugs while undergoing treatment with effective psychological interventions, which are used to improve their drug avoidance behavior $(11,12)$. A good treatment strategy aims to reduce the craving for drugs and prevent relapse, and both of these treatments have shown modest effects in reducing the occurrence of craving and other related behaviors $(11,12)$. However, as a long-acting synthetic opioid drug, emerging evidence suggests that methadone may have negative consequences for the integrity of brain white matter and brain metabolism and function when used for long periods of time (13). Meanwhile, the effect of longterm MMT on cue-induced craving and brain activation remains poorly understood.

Reducing craving behavior is at the core of all addiction therapy strategies. Cue-induced drug cravings play an important role underlying relapse. Conditioned cues can elicit drug-seeking behavior, even after prolonged periods of abstinence (14), and dysfunction of the mesolimbic, visuospatial-attention, prefrontal cortex, and cerebellum regions are shown to be involved in heroin-related cues (15-21). Using an event-related functional magnetic resonance imaging (fMRI) paradigm, our previous studies found that short-term MMT patients demonstrated higher drug-cuerelated brain activation than those undergoing PA (22), and that HUD patients showed decreased brain activation after approximately 6 months of abstinence when compared to 1 month of abstinence (17). We also found that longterm MMT can reduce former heroin users' induced response of the bilateral dorsal striatum to heroin cues (18). These results suggested that during abstinence periods of different lengths, MMT and PA patients have different brain activation responses to drug cues; both MMT and PA patients have higher drug-cue-related responses during short-term treatment, whereas with long-term treatment, PA patients show reduced brain activation. However, the difference between long-term MMT and PA is still unclear.

The present study aimed to identify the difference in brain activation induced by heroin cues between longterm MMT and PA patients using an event-related fMRI paradigm. We hypothesized that long-term MMT may lead to adaptive functional changes in some regions related to heroin-cue exposure, and that long-term PA is helpful in reducing brain activation and subjective craving related to heroin cues.

\section{Methods}

\section{Subjects}

In total, 71 participants were recruited. Twenty-five HUD patients undergoing outpatient long-term PA were recruited from the Drug Rehabilitation Center in Lantian, Xi'an, and 24 HUD patients receiving outpatient long-term MMT were recruited from the Baqiao MMT clinic, Xi'an. A control group comprising 22 healthy controls (HCs) from the local district around Tangdu Hospital was also recruited. The inclusion criteria for HUD subjects included: (I) meeting the diagnostic criteria of DSM-V-TR (Diagnostic and Statistical Manual of Mental Disorders, Fifth Edition) for HUD; (II) undergoing PA or MMT for approximately 12 months; and (III) right handed. The exclusion criteria included: (I) abuse of an illegal drug other than heroin; (II) neurological signs and/or a history of neurological disease; (III) current chronic medical illness; (IV) daily consumption of alcohol; (V) a history of head trauma; (VI) cardiovascular, pulmonary, or systemic disease; (VII) claustrophobia; and (VIII) any contraindications for MRI. All subjects were cigarette smokers; to avoid the acute effects of cigarette smoking on the brain, we required all subjects to refrain from smoking for 24 hours prior to the start of the MRI 
scan. HCs were included if they had no history of substance abuse or neuropsychiatric disorder.

\section{Design and procedure}

This study adopted an event-related design (15-19). Twenty-four heroin-cue pictures and 24 neutral pictures were used. Heroin-cue pictures included heroin injection, paraphernalia, and preparation, and neutral pictures included household objects or chores (Figure S1). The pictures were projected onto a mirror fixed on the head coil in a pseudo-randomized order with E-Prime 2.0 software (Psychology Software Tools, Inc., Pittsburgh, PA, USA). The pictures were presented for 2 seconds with a variable inter-stimulus interval ranging from 4-12 seconds (mean $=8$ seconds), during which a crosshair was presented. The entire task was completed in 1 session and lasted 490 seconds (Figure S2). To avoid the acute effect of methadone, the fMRI scan was conducted at least 5-8 hours after daily methadone use (8). Consumption of any tea, alcohol, caffeine, or medicine was prohibited within the 12 hours before the scan. A $0-10$ visual analog scale was used to assess subjective heroin cravings in the HUD patients (15-19). Craving ratings were acquired before and shortly after each fMRI scan. The HUD patients were given a debriefing interview after the fMRI scan to reduce the influence of the heroin-cue exposure.

As co-morbidity of drug addiction with psychiatric disorders is widely reported, the Beck Depression Inventory II (BDI) and the Hamilton Anxiety Scale (HAMA) were used to assess the presence of co-morbid depression and anxiety.

\section{MRI data acquisition}

All imaging data were acquired on a 3.0 T GE-Signa Excite HD MRI scanner (GE Healthcare, Milwaukee, USA). $T_{2}$-weighted images ( $\mathrm{T}_{2} \mathrm{WIs}$ ) were collected and checked by an experienced radiologist to exclude any gross structural abnormality. Gradient Echo Planar Imaging was used for the collection of the fMRI images (repetition time $=2,000$ milliseconds, echo time $=30$ milliseconds, flip angle $=90^{\circ}$, matrix $=64 \times 64$, field of view $=256 \times 256 \mathrm{~mm}^{2}$, slice thickness $=4 \mathrm{~mm}$, gap = $0 \mathrm{~mm}$, spatial resolution $\left.=4 \times 4 \times 4 \mathrm{~mm}^{3}\right)(22)$.

\section{Data preprocessing}

The Processing Assistant for Resting-State fMRI (DPARSF) software was used for the data processing. The fMRI data analysis was conducted using Statistical Parametric Mapping 12 software (SPM12) (http://www.fil.ion.ucl. ac.uk/spm). The data preprocessing was performed in the same manner as that in our previous study (22). First, fMRI images were slice-time-corrected with reference to the first slice, corrected for motion, registered to the highresolution 3-dimensional $T_{1}$-weighted images ( $\left.T_{1} W I s\right)$, and then normalized to a standard SPM $T_{1}$ template. Subjects with head motion of more than $1.5 \mathrm{~mm}$ in translation or $1.5^{\circ}$ in rotation were excluded. A statistical model for each subject was computed by applying a canonical response function. Regionally specific condition effects were tested by employing linear contrasts for each subject and different conditions. The critical contrast of interest was the heroinrelated versus neutral cues, which would reveal brain activity related to the processing of heroin-related cues (23). Correction for multiple comparisons was conducted using DPABI software (http://rfmri.org/DPABI)via the Gaussian Random Field (GRF) Theory correction program within the whole brain (voxel $\mathrm{P}<0.001$, cluster $\mathrm{P}<0.05$, 2-tailed).

A region of interest (ROI)-based correlation analysis was performed on all HUD patients to assess the relationship between changes in cravings and brain activation. Peak coordinate voxels of each differential cluster were defined as centers for the sphere-shaped ROIs (radius $=3 \mathrm{~mm}$ ). Changes in brain activation within the ROIs of the HUD subjects were extracted and their relationships with the changes in craving were identified through Pearson's correlation analysis. Statistical significance was set as $\mathrm{P}<0.05$ (corrected by false discovery rate).

\section{Results}

\section{Demographic and behavioral characteristics}

Six participants were excluded due to excessive head motion (2 MMT, $1 \mathrm{PA}$, and $1 \mathrm{HC}$ ) and gross brain abnormality in $\mathrm{T}_{2}$ WIs (1 MMT and $1 \mathrm{HC}$ ). Therefore, $24 \mathrm{PA}$ patients, 21 MMT patients, and $20 \mathrm{HCs}$ were included in the final analysis. As presented in Table 1, there were no statistical differences in age, education, or cigarette use among the 3 groups. No significant difference was found in heroin use history or duration of therapy in the HUD patients. However, there were significant differences in the BDI and HAMA scores among the 3 groups. Compared with the HCs, PA patients had significantly increased depression and anxiety scores, and MMT patients had significantly 
Table 1 Demographic and clinical characteristics of participants

\begin{tabular}{|c|c|c|c|c|c|}
\hline Characteristics & $\mathrm{HC}(\mathrm{n}=20)$ & MMT (n=21) & PA $(n=24)$ & \multicolumn{2}{|c|}{ Group differences } \\
\hline Age (years) & $35.2 \pm 7.0$ & $35.1 \pm 9.3$ & $33.0 \pm 6.8$ & $F=0.589$ & 0.558 \\
\hline Years of education & $10.2 \pm 2.3$ & $9.2 \pm 1.7$ & $10.3 \pm 3.1$ & $F=1.305$ & 0.279 \\
\hline Duration of heroin use (months) & NA & $57.8 \pm 66.5$ & $78.8 \pm 50.4$ & $t=1.179$ & 0.246 \\
\hline Total heroin dose (g) & NA & $510.9 \pm 495.2$ & $1,880.2 \pm 2,105.7$ & $t=1.559$ & 0.061 \\
\hline Average methadone dose (mg/day) & NA & $43.6 \pm 18.5$ & NA & NA & NA \\
\hline Total methadone dose $(\mathrm{mg})$ & NA & $15,109.6 \pm 7,024.7$ & NA & NA & NA \\
\hline Duration of MMT/PA (months) & NA & $11.5 \pm 2.1$ & $11.8 \pm 1.4$ & $t=0.685$ & 0.498 \\
\hline
\end{tabular}

$\mathrm{HC}$, healthy control; MMT, methadone maintenance treatment; PA, protracted abstinence; NA, not available.

Table 2 BDI and HAMA Scores in the PA, MMT, and HC groups

\begin{tabular}{lcccc}
\hline \multirow{2}{*}{ Items } & P value (from ANOVA) & & \multicolumn{2}{c}{ Post Hoc/P value } \\
\cline { 3 - 5 } & $0.000^{*}$ & HC vs. PA & HC vs. MMT & $0.047^{*}$ \\
BDI Score & $0.000^{*}$ & $0.000^{*}$ & 0.257 & $0.029^{*}$
\end{tabular}

*, $\mathrm{P}<0.05$, statistically significant difference. ANOVA, analysis of variance; BDI, Beck Depression Inventory; HAMA, Hamilton Anxiety Rating Scale; HC, healthy control; MMT, methadone maintenance treatment; PA, protracted abstinence.

Table 3 Subjective craving scores in the PA and MMT groups

\begin{tabular}{lcccc}
\hline Characteristics & PA & MMT & $\mathrm{t}$ & $\mathrm{p}$ \\
\hline Before cue exposure & $1.05 \pm 1.12$ & $3.04 \pm 2.44$ & 3.44 & $0.001^{*}$ \\
After cue exposure & $2.00 \pm 1.97$ & $4.33 \pm 3.15$ & 2.92 & $0.006^{*}$ \\
Change in craving & $1.00 \pm 1.45$ & $1.29 \pm 1.57$ & 0.65 & 0.52 \\
\hline
\end{tabular}

*, $\mathrm{P}<0.05$, statistically significant difference. MMT, methadone maintenance treatment; PA, protracted abstinence.

increased depression scores. Compared with the MMT patients, those undergoing PA had significantly increased anxiety scores (Table 2).

\section{Subjective craving}

Both before and after heroin-cue exposure, MMT patients showed significantly higher subjective craving than PA patients, although there was no statistical difference in the change in subjective craving between the 2 groups (Table 3).

\section{Imaging results}

The PA, MMT, and HC groups showed significant differences in heroin cue-induced brain activation. Compared to the HCs, the MMT and PA groups demonstrated significantly higher brain activation in the left pallidum, middle occipital gyrus, postcentral gyrus, anterior cingulate cortex (ACC), middle cingulate cortex (MCC), inferior parietal lobule (IPL), superior parietal lobule (SPL), amygdala, hippocampus, right inferior temporal gyrus, 


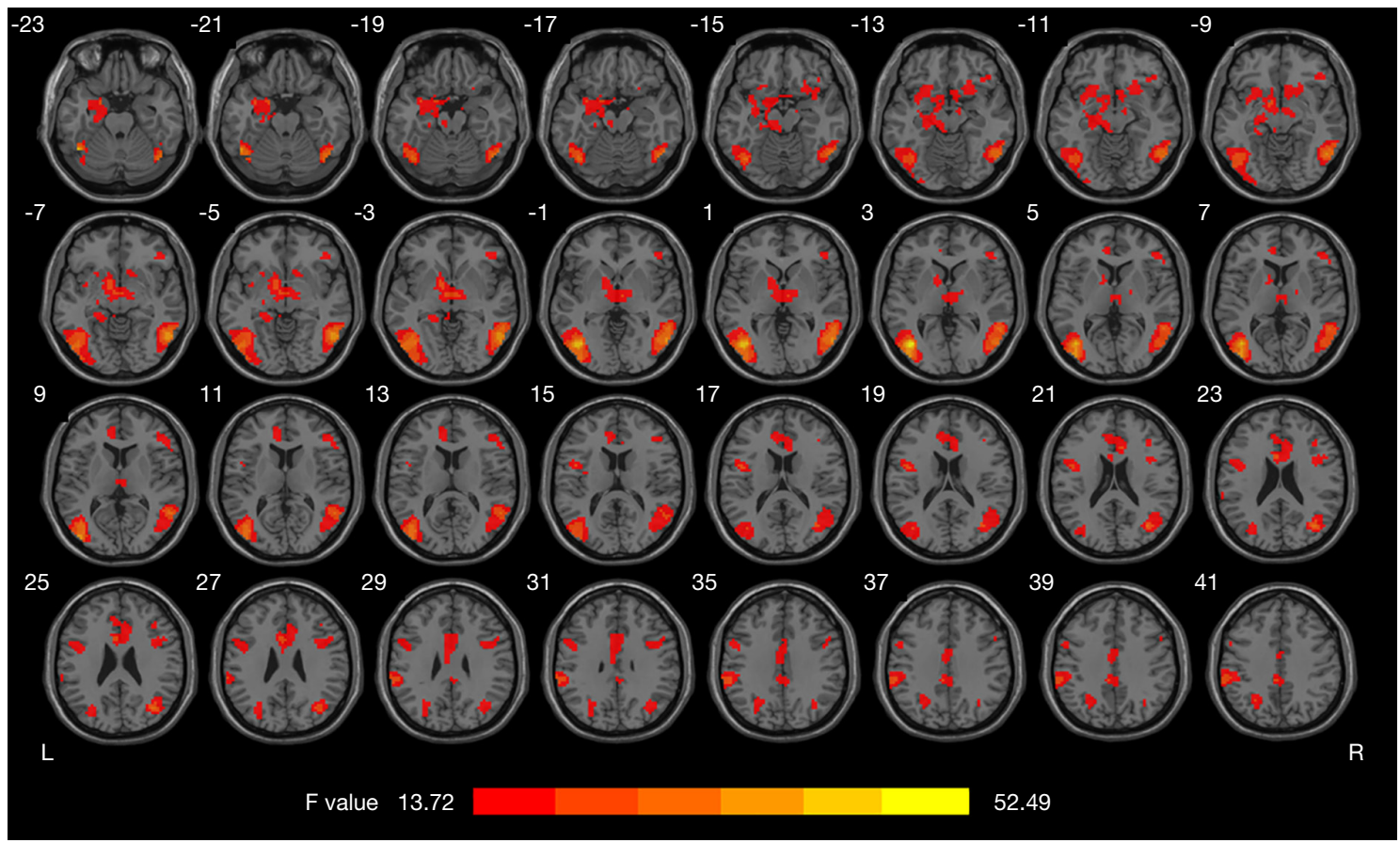

Figure 1 Analysis of variance results of the differences in brain activation during exposure to heroin-related cues in the MMT, PA, and HC groups ( $\mathrm{P}<0.05$, corrected for GRF simulations; L: left, R: right). HC, healthy control; MMT, methadone maintenance treatment; PA, protracted abstinence.

inferior frontal gyrus triangularis (IFG-triang), and caudate (Figure 1, Table 4). Compared with the HCs, MMT patients showed significantly higher heroin-cue-induced brain activation in all of the regions, including the mesolimbic region (left hippocampus, cingulate cortex, and amygdala), reward-related region (right caudate and left pallidum), visuospatial-attention region (right IFG-triang, left SPL, left IPL, and left middle occipital gyrus), somatosensory cortex (left postcentral gyrus), and auditory cortex region (right inferior temporal gyrus). No difference was found in brain activation between the HC and MMT groups. MMT patients also showed significantly higher heroin-cueinduced brain activation in all of the regions mentioned above relative to the PA group. Compared with the HCs, the PA groups showed significantly higher heroin-cue-induced brain activation in the left IPL and left SPL (Figure 2).

\section{Correlation}

The relationship between the changes in craving and changes in brain activation intensity was analyzed; however, no correlation results survived multiple comparison correction.

\section{Discussion}

The present study attempted to investigate the difference in heroin-cue-related brain activation between individuals undergoing long-term PA and MMT, in order to identify which treatment strategy is more conducive to reducing the heroin-cue-induced craving response so as to be beneficial in rehabilitating brain function. Several different treatment methods exist for different addictive substances. For instance, a previous study reported on the immediate effects of acupuncture on craving after tobacco cessation based on the fractional amplitude of low-frequency fluctuation (fALFF) (24). Our research used event-related fMRI and found that although patients receiving long-term MMT complied with the treatment, they still reported relatively higher subjective cravings and demonstrated higher heroincue-induced brain activation than PA patients. In contrast, long-term PA for HUD patients appears to be more beneficial than MMT in reducing subjective cravings and in lowering the salience value of drug-related cues.

In 2008, "The People's Republic of China Anti-Drug Law" extended the time of PA from 3-6 months to 1-3 years. It also defined long-term abstinence for HUD patients 
Table 4 Activated brain regions in the 3 groups in response to heroin-related $v$ s. neutral cues

\begin{tabular}{|c|c|c|c|c|c|c|c|}
\hline Brain regions & Left (L)/right (R) & Brodmann's area & \multicolumn{3}{|c|}{ Peak location } & Peak $F_{2,62}$ score & Voxel numbers \\
\hline Pallidum & $\mathrm{L}$ & 25 & -12 & 3 & -5 & 22.35 & 830 \\
\hline Middle occipital gyrus & $\mathrm{L}$ & 37 & -42 & -69 & 3 & 52.49 & 1450 \\
\hline Inferior temporal gyrus & $\mathrm{R}$ & 37 & 54 & -60 & -6 & 36.24 & 890 \\
\hline Postcentral gyrus & $L$ & 48 & -45 & 0 & 21 & 19.25 & 135 \\
\hline ACC & $\mathrm{L}$ & - & -3 & 12 & 27 & 19.76 & 320 \\
\hline MCC & $\mathrm{L}$ & 23 & -3 & -36 & 39 & 16.79 & 58 \\
\hline IPL & L & 7 & -33 & -56 & 50 & 17.98 & 82 \\
\hline Caudate & $\mathrm{R}$ & 25 & 12 & 14 & -10 & 12.35 & 82 \\
\hline Hippocampus & $\mathrm{L}$ & - & -22 & -21 & -11 & 11.22 & 40 \\
\hline
\end{tabular}

ACC, anterior cingulate cortex; IFG-triang, inferior frontal gyrus triangularis; IPL, inferior parietal lobule; MCC, middle cingulate cortex; SPL, superior parietal lobule.

as more than 1 year of PA treatment. The main purposes of the treatment are to improve the curative effect and to prevent heroin relapse, although the effects of long-term treatment and the potential neuromechanisms involved remain unclear. In our previous study, we found that HUD patients who received short-term MMT (for approximately 6 months) demonstrated higher heroin-cue-induced activation in the mesolimbic, visuo-spatial attention regions, memory retrieval related regions, and somatosensory cortex compared to those receiving short-term PA (22). In the present study, long-term MMT patients continued to show significantly higher heroin-cue-induced brain activation, mainly in the mesolimbic, reward-related, and visuospatialattention regions.

The limbic system plays an important role in the regulation of memory and emotion. We found heroincue-induced activation to be significantly increased in the amygdala, cingulate cortex, and hippocampus of longterm MMT patients when compared to that of the PA and $\mathrm{HC}$ subjects. The cingulate cortex is one of the brain regions most affected by heroin addiction. Ventral ACC dysfunction may lead to drug-cue reactivity and decisionmaking deficits, and affects the encoding of reward expectations. The rostral part of the ACC is responsible for inhibitory control (25), and in HUD patients, it shows decreased functional connectivity with the orbital frontal gyrus, dorsolateral prefrontal lobe, and right temporal lobe, which manifests as impaired inhibition and control over decision-making (26). Some studies have indicated that the ACC plays a significant role in behavioral regulation and emotional responses, including reward and punishment $(27,28)$, and that emotional dysregulation is the initiating factor for relapse (29). In addition to the ACC, this study also found that the MCC showed increased activation in response to heroin cues. We also found that MMT patients had a higher subjective craving than the PA patients both before and after exposure to cues. The potential relationship between the higher subjective craving and significantly increased activation in the cingulate cortex in MMT patients should be investigated in future studies.

The amygdala is responsible for the acquisition, consolidation, and expression of drug-cue learning, which can induce cravings for drugs and drive relapse (30). One study reported that memory-related brain regions, such as the amygdala and hippocampus, were activated when drugdependent individuals were exposed to drug cues (31), which is similar to our present findings. The amygdala also plays an important role in reward processing, and a previous study found that when smokers were exposed to smoking-related stimuli, the activation of the amygdala was 

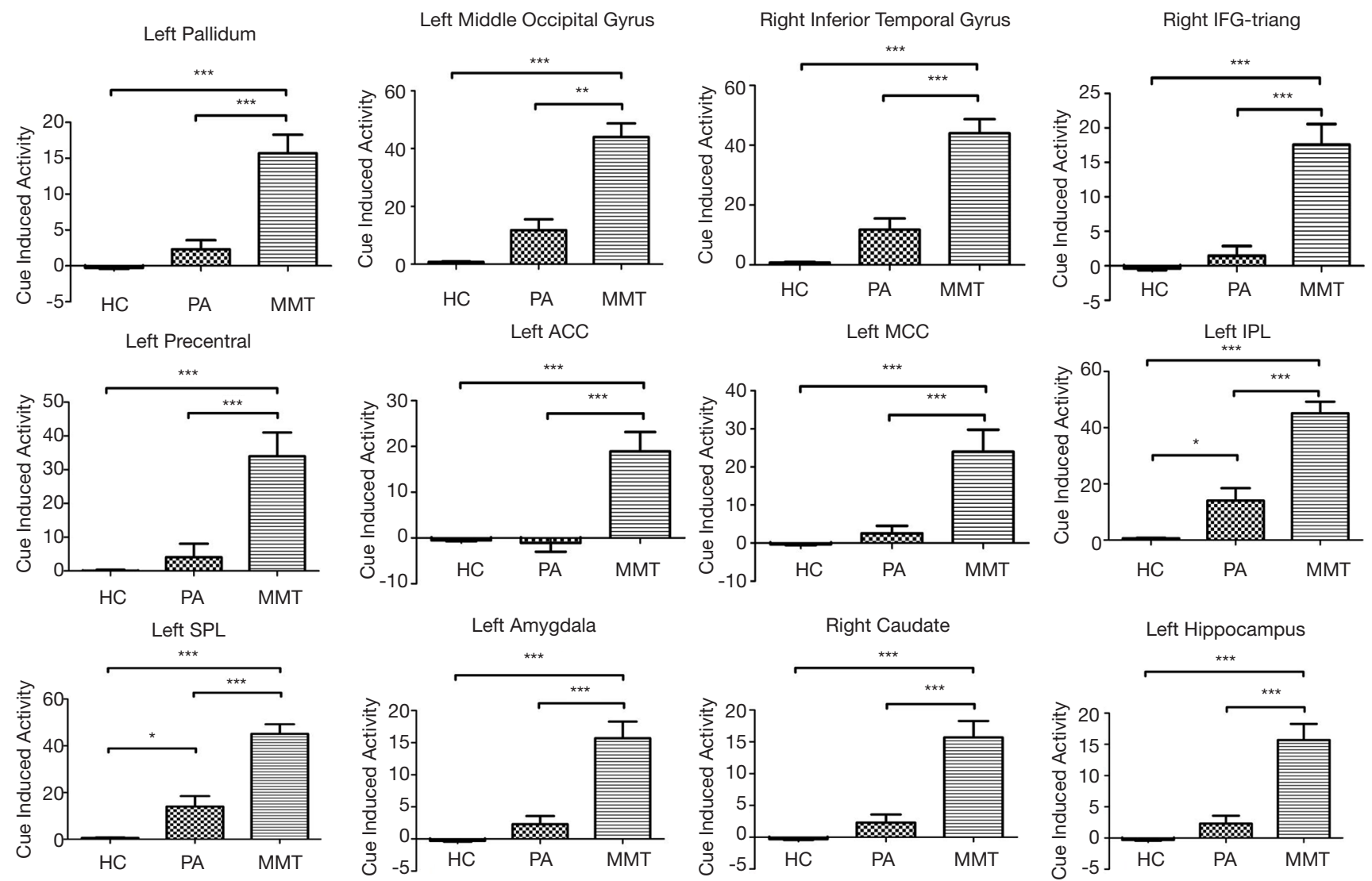

Figure 2 Post hoc analysis shows a difference in signal change in response to 'heroin > neutral cues' contrast among MMT, PA, and HC groups. ${ }^{*} \mathrm{P}<0.05,{ }^{* *} \mathrm{P}<0.01,{ }^{* * *} \mathrm{P}<0.001$. HC, healthy control; MMT, methadone maintenance treatment; PA, protracted abstinence; IFGtriang, inferior frontal gyrus triangularis; ACC, anterior cingulate cortex; MCC, middle cingulate cortex; SPL, superior parietal lobule; IPL, inferior parietal lobule.

associated with a smoker's level of craving (32). In this study, we found that MMT patients have significantly higher heroin-cue-induced activation in the limbic region, which may be related to the specific distribution of methadone in the brain. Methadone, as a synthetic opioid, is well absorbed in the brain regions rich in $\mu$-opioid receptors (33), which are predominately located in the limbic system in humans $(34,35)$. Although the relationship between the specific distribution of methadone in the limbic system and the increase of drug-cue-induced activity requires further confirmation, we believe that MMT patients remain at a higher risk of relapse caused by heroin-related cues.

The reward circuit consists of the nucleus accumbens and ventral pallidum. When a heroin user is stimulated by cues, the brain's response is affected by the expected reward. The dopamine neurons projected into the reward circuit increase the secretion of dopamine, which is in direct proportion to the individual's level of euphoria (36). The stronger the expectation of reward from the cue, the greater the motivation and craving to obtain the drug. This explains why the heroin-cue response in the pallidum was significantly enhanced in the MMT and PA groups compared to in the HC group, and is likely due to the stronger cravings experienced by individuals with HUD. The posterior central gyrus plays an extremely important role in emotional management and visual recognition, and is part of the somatosensory motor cortex (37). Increased activation in the sensory and motor cortex has been shown to be related to drug-cue-related relapse in cocaine abuse patients (38). In the present study, the higher activation in the left central posterior gyrus in long-term MMT patients may reflect the potential for drug-cue-related relapse risk during MMT treatment, which is in line with the higher relapse rate in MMT patients. 
Our post-hoc analysis results showed that, except for the left IPL and SPL regions related to visuospatial attention, there were no statistical differences between the long-term PA group and the HCs. This finding is in contrast with that of our previous study in which short-term PA patients showed extensive heroin-cue-induced brain activation compared to the HCs (22). After long-term treatment in this study, the reduced heroin-cue-related brain activation may reflect the partial recovery of the abnormal brain function. Rapeli et al. reported that the therapeutic effect of abstinence is related to its duration, and they further showed that the duration of abstinence may affect the change in the individual's cognitive ability (39), which is in line with the partial recovery of heroin-induced dysfunction observed in the PA group in the present study. It should be noted that significantly increased depression and anxiety symptoms were detected in long-term PA patients in this study, and depression and anxiety have previously been identified as important factors for relapse following a period of drug abstinence in HUD patients (40). Consequently, although long-term PA contributes to the recovery of brain function, patients with high anxiety and depressive symptoms should be closely monitored, and effective behavioral interventions should be implemented.

There are several limitations to this study. Firstly, only males were included in the study, and therefore possible sexrelated differences between the 2 strategies may not have been identified. Secondly, due to poor compliance, a high loss rate, and difficulty in recruiting HUD patients, this study is cross-sectional by design. A longitudinal design will be considered for future experiments. Thirdly, the detection of heroin relapse through a monthly fixed urine just a rough estimate, the more exact method should be used in future design. Although the patients in the study cooperated well, they were all outpatients, and therefore the accuracy of their heroin usage cannot be guaranteed.

\section{Conclusions}

In summary, long-term PA appears to be more beneficial than MMT in lowering the salience value of drug-related cues in HUD patients, although the higher anxiety and depressive symptoms reported during PA treatment should be of concern. Although MMT is regarded as the most effective treatment for HUD, long-term MMT patients have higher subjective craving and heroin-cue-induced activation located mainly in the mesolimbic, reward-related, and visuospatial-attention regions.

\section{Acknowledgments}

We thank Mr. Xinhai Wu for his efforts in the recruitment of the heroin use disorder patients.

Funding: This study was funded by the National Natural Science Foundation of China (81771813, 81401393 and $81671661)$, the Technology Innovation Development Foundation of Tangdu Hospital (2013LCYJ003), the China Postdoctoral Science Foundation (2020M680607), and the Research Foundation of Beijing Friendship Hospital, Capital Medical University (yyqdkt2019-31).

\section{Footnote}

Conflicts of Interest: All authors have completed the ICMJE uniform disclosure form (available at http://dx.doi. org/10.21037/qims-20-1002). The authors have no conflicts of interest to declare.

Ethical Statement: This study was approved by the ethics committee of Tangdu Hospital (TDLL-2017008). Written informed consent was obtained from all participants included in the study.

Open Access Statement: This is an Open Access article distributed in accordance with the Creative Commons Attribution-NonCommercial-NoDerivs 4.0 International License (CC BY-NC-ND 4.0), which permits the noncommercial replication and distribution of the article with the strict proviso that no changes or edits are made and the original work is properly cited (including links to both the formal publication through the relevant DOI and the license). See: https://creativecommons.org/licenses/by-nc-nd/4.0/.

\section{References}

1. Koob GF, Volkow ND. Neurobiology of addiction: a neurocircuitry analysis. Lancet Psychiatry 2016;3:760-73.

2. Xiao Z, Lee T, Zhang JX, Wu Q, Wu R, Weng X, Hu X. Thirsty heroin addicts show different $\mathrm{fMRI}$ activations when exposed to water-related and drug-related cues. Drug Alcohol Depend 2006;83:157-62.

3. Tang YL, Zhao D, Zhao C, Cubells JF. Opiate addiction in China: current situation and treatments. Addiction 2006;101:657-65.

4. Volkow ND, Blanco C. The changing opioid crisis: development, challenges and opportunities. Mol Psychiatry 2021;26:218-33. 
5. O'Brien CP. A range of research-based pharmacotherapies for addiction. Science 1997;278:66-70.

6. Dackis CA, O'Brien CP. Cocaine dependence: a disease of the brain's reward centers. J Subst Abuse Treat 2001;21:111-7.

7. Fernando A. Wagner JCA. Into the World of Illegal Drug Use: Exposure Opportunity and Other Mechanisms Linking the Use of Alcohol, Tobacco, Marijuana, and Cocaine. Am J Epidemiol 2002;166:918-25.

8. Kreek MJ. Methadone-related opioid agonist pharmacotherapy for heroin addiction. History, recent molecular and neurochemical research and future in mainstream medicine. Ann N Y Acad Sci 2000;909:186-216.

9. Wei X, Wang L, Wang X, Li J, Li H, Jia W. A study of 6-year retention in methadone maintenance treatment among opioid-dependent patients in Xi'an. J Addict Med 2013;7:342-8.

10. Bratberg JP. Opioids, naloxone, and beyond: The intersection of medication safety, public health, and pharmacy. J Am Pharm Assoc (2003) 2017;57:S5-S7.

11. Hser YI, Douglas AM, Fletcher B. Comparative Treatment Effectiveness Effects of Program Modality and Client Drug Dependence History on Drug Use Reduction. J Subst Abuse Treat 1998;15:513-23.

12. Prendergast ML, Podus D, Chang E. Program Factors and Treatment Outcomes in Drug Dependence Treatment: An Examination Using Meta-Analysis. Subst Use Misuse 2000;35:1931-65.

13. Li W, Li Q, Wang Y, Zhu J, Ye J, Yan X, Li Y, Chen J, Liu J, Li Z, Wang W, Liu Y. Methadone-induced Damage to White Matter Integrity in Methadone Maintenance Patients: A Longitudinal Self-control DTI Study. Sci Rep 2016;6:19662.

14. Grimm JW, Harkness JH, Ratliff C, Barnes J, North K, Collins S. Effects of systemic or nucleus accumbensdirected dopamine D1 receptor antagonism on sucrose seeking in rats. Psychopharmacology (Berl) 2011;216:219-33.

15. Wang W, Li Q, Wang Y, Tian J, Yang W, Li W, Qin W, Yuan K, Liu J. Brain fMRI and craving response to heroin-related cues in patients on methadone maintenance treatment. Am J Drug Alcohol Abuse 2011;37:123-30.

16. Li Q, Wang Y, Zhang Y, Li W, Yang W, Zhu J, Wu N, Chang H, Zheng Y, Qin W, Zhao L, Yuan K, Liu J, Wang W, Tian J. Craving correlates with mesolimbic responses to heroin-related cues in short-term abstinence from heroin: an event-related fMRI study. Brain Res 2012;1469:63-72.
17. Li Q, Wang Y, Zhang Y, Li W, Zhu J, Zheng Y, Chen J, Zhao L, Zhou Z, Liu Y, Wang W, Tian J. Assessing cue-induced brain response as a function of abstinence duration in heroin-dependent individuals: an event-related fMRI study. PLoS One 2013;8:e62911.

18. Wang Y, Wang H, Li W, Zhu J, Gold MS, Zhang D, Wang L, Li Y, Yan X, Cheng J, Li Q, Wang W. Reduced responses to heroin-cue-induced craving in the dorsal striatum: effects of long-term methadone maintenance treatment. Neurosci Lett 2014;581:120-4.

19. Li Q, Li W, Wang H, Wang Y, Zhang Y, Zhu J, Zheng Y, Zhang D, Wang L, Li Y, Yan X, Chang H, Fan M, Li Z, Tian J, Gold MS, Wang W, Liu Y. Predicting subsequent relapse by drug-related cue-induced brain activation in heroin addiction: an event-related functional magnetic resonance imaging study. Addict Biol 2015;20:968-78.

20. Daglish MR, Weinstein A, Malizia AL, Wilson S, Melichar JK, Britten S, Brewer C, Lingford-Hughes A, Myles JS, Grasby P, Nutt DJ. Changes in regional cerebral blood flow elicited by craving memories in abstinent opiatedependent subjects. Am J Psychiatry 2001;158:1680-6.

21. Zijlstra F, Veltman DJ, Booij J, van den Brink W, Franken IH. Neurobiological substrates of cue-elicited craving and anhedonia in recently abstinent opioid-dependent males. Drug Alcohol Depend 2009;99:183-92.

22. Wei X, Li W, Chen J, Li Y, Zhu J, Shi H, Liu J, Xue J, Liu W, Wang F, Liu Y, Dang S, Chen J, Li Q, Wang $W$. Assessing drug cue-induced brain response in heroin dependents treated by methadone maintenance and protracted abstinence measures. Brain Imaging Behav 2020;14:1221-9.

23. Franklin TR, Wang Z, Wang J, Sciortino N, Harper D, Li Y, Ehrman R, Kampman K, O'Brien CP, Detre JA, Childress AR. Limbic activation to cigarette smoking cues independent of nicotine withdrawal: a perfusion fMRI study. Neuropsychopharmacology 2007;32:2301-9.

24. Wang YY, Liu Z, Chen F, Sun L, Wu Y, Yang JS, Fang JL. Effects of acupuncture on craving after tobacco cessation: a resting-state $\mathrm{fMRI}$ study based on the fractional amplitude of low-frequency fluctuation. Quant Imaging Med Surg 2019;9:1118-25.

25. Liu J, Liang J, Qin W, Tian J, Yuan K, Bai L, Zhang Y, Wang W, Wang Y, Li Q, Zhao L, Lu L, von Deneen KM, Liu Y, Gold MS. Dysfunctional connectivity patterns in chronic heroin users: an fMRI study. Neurosci Lett 2009;460:72-7.

26. Yuan K, Qin W, Dong M, Liu J, Liu P, Zhang Y, Sun J, Wang W, Wang Y, Li Q, Yang W, Tian J. Combining 
spatial and temporal information to explore restingstate networks changes in abstinent heroin-dependent individuals. Neurosci Lett 2010;475:20-4.

27. Kelly AM, Di Martino A, Uddin LQ, Shehzad Z, Gee DG, Reiss PT, Margulies DS, Castellanos FX, Milham MP.

Development of anterior cingulate functional connectivity from late childhood to early adulthood. Cereb Cortex 2009; 19:640-57.

28. Phan KL, Fitzgerald DA, Nathan PJ, Moore GJ, Uhde TW, Tancer ME. Neural substrates for voluntary suppression of negative affect: a functional magnetic resonance imaging study. Biol Psychiatry 2005;57:210-9.

29. Berking M, Margraf M, Ebert D, Wupperman P, Hofmann SG, Junghanns K. Deficits in emotion-regulation skills predict alcohol use during and after cognitive-behavioral therapy for alcohol dependence. J Consult Clin Psychol 2011;79:307-18.

30. Ma N, Liu Y, Li N, Wang CX, Zhang H, Jiang XF, $\mathrm{Xu} \mathrm{HS}, \mathrm{Fu} \mathrm{XM}, \mathrm{Hu} \mathrm{X}$, Zhang DR. Addiction related alteration in resting-state brain connectivity. Neuroimage 2010;49:738-44.

31. Kilts CD, Schweitzer JB, Quinn CK, Gross RE, Faber TL, Muhammad F, Ely TD, Hoffman JM, Drexler KP. Neural activity related to drug craving in cocaine addiction. Arch Gen Psychiatry 2001;58:334-41.

32. Wang Z, Faith M, Patterson F, Tang K, Kerrin K, Wileyto EP, Detre JA, Lerman C. Neural substrates of abstinenceinduced cigarette cravings in chronic smokers. J Neurosci 2007;27:14035-40.

33. Corliss RF, Mandal R, Soriano BJ. Bilateral acute necrosis

Cite this article as: Wei X, Li Q, Chen J, Shen B, Wang W, Li W. Differences in cue-induced brain activation between long-term methadone maintenance treatment and protracted abstinence in heroin use disorder patients: a functional magnetic resonance imaging study. Quant Imaging Med Surg 2021;11(5):2104-2113. doi: 10.21037/qims-20-1002 of the globi pallidi and rhabdomyolysis due to combined methadone and benzodiazepine toxicity. Am J Forensic Med Pathol 2013;34:1-4.

34. Kuhar MJ, Pert CB, Snyder SH. Regional distribution of opiate receptor binding in monkey and human brain. Nature 1973;245:447-50.

35. Pertschuk LP, Sher JH, Ford DH. A morphological comparison of the regional distribution of methadone in human and rat brain as demonstrated by immunofluorescence. Drug Alcohol Depend 1976;1:247-54.

36. Volkow ND, Li TK. Drug addiction: the neurobiology of behaviour gone awry. Nat Rev Neurosci 2004;5:963-70.

37. Adolphs R, Damasio H, Tranel D, Cooper G, Damasio AR. A role for somatosensory cortices in the visual recognition of emotion as revealed by three-dimensional lesion mapping. J Neurosci 2000;20:2683-90.

38. Kosten TR, Scanley BE, Tucker KA, Oliveto A, Prince C, Sinha R, Potenza MN, Skudlarski P, Wexler BE. Cue-induced brain activity changes and relapse in cocaine-dependent patients. Neuropsychopharmacology 2006;31:644-50.

39. Rapeli P, Kivisaari R, Autti T, Kahkonen S, Puuskari V, Jokela O, Kalska H. Cognitive function during early abstinence from opioid dependence: a comparison to age, gender, and verbal intelligence matched controls. BMC Psychiatry 2006;6:9.

40. Conner KR, Pinquart M, Duberstein PR. Meta-analysis of depression and substance use and impairment among intravenous drug users (IDUs). Addiction 2008;103:524-34. 

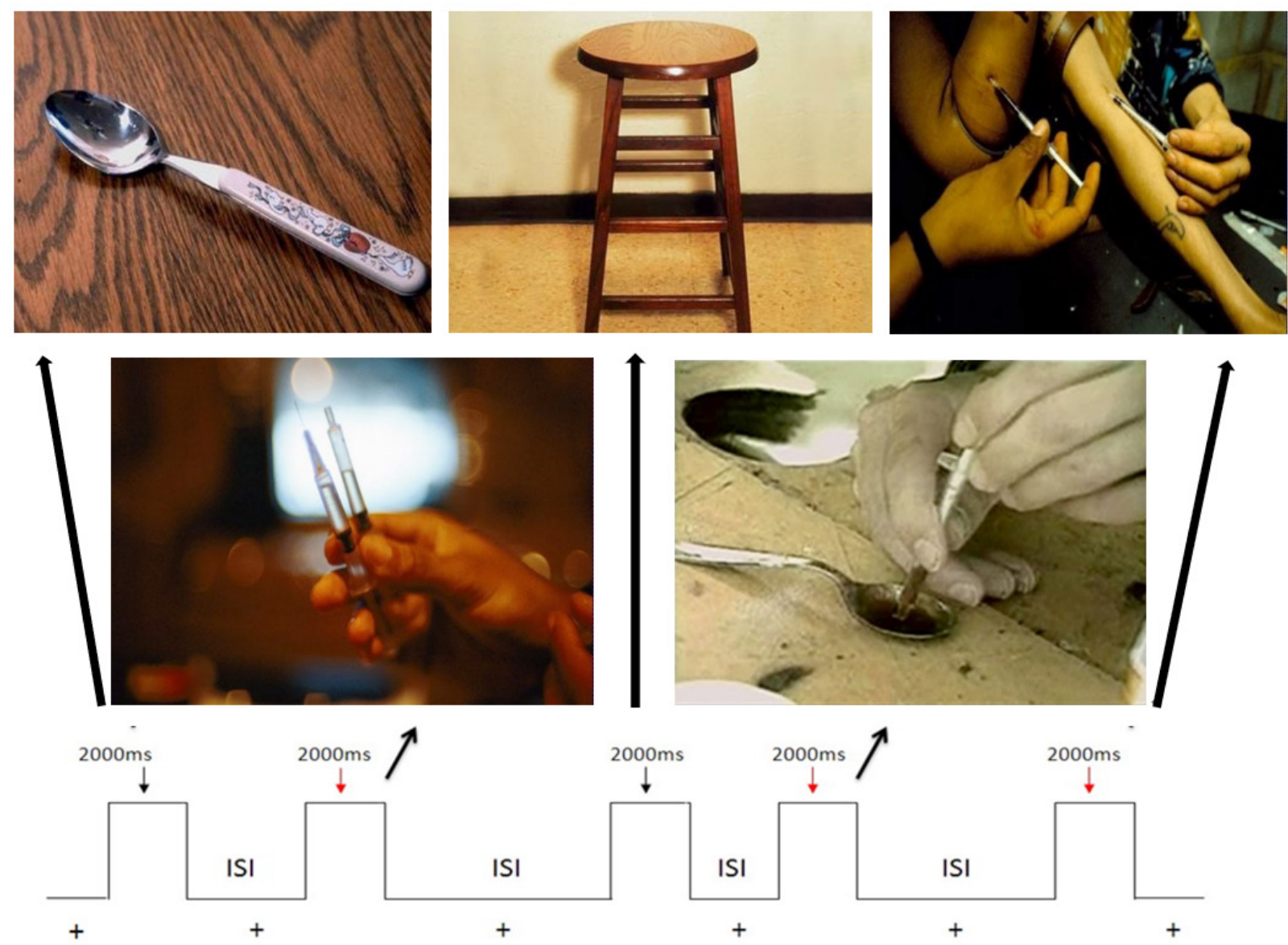

Figure S1 An example of the event-related drug-cue response functional magnetic resonance imaging task. There are 2 types of image cues, including 24 heroin-related and 24 neutral image cues. The former includes images of heroin injection, paraphernalia, and preparation, and the latter includes household objects or chores.

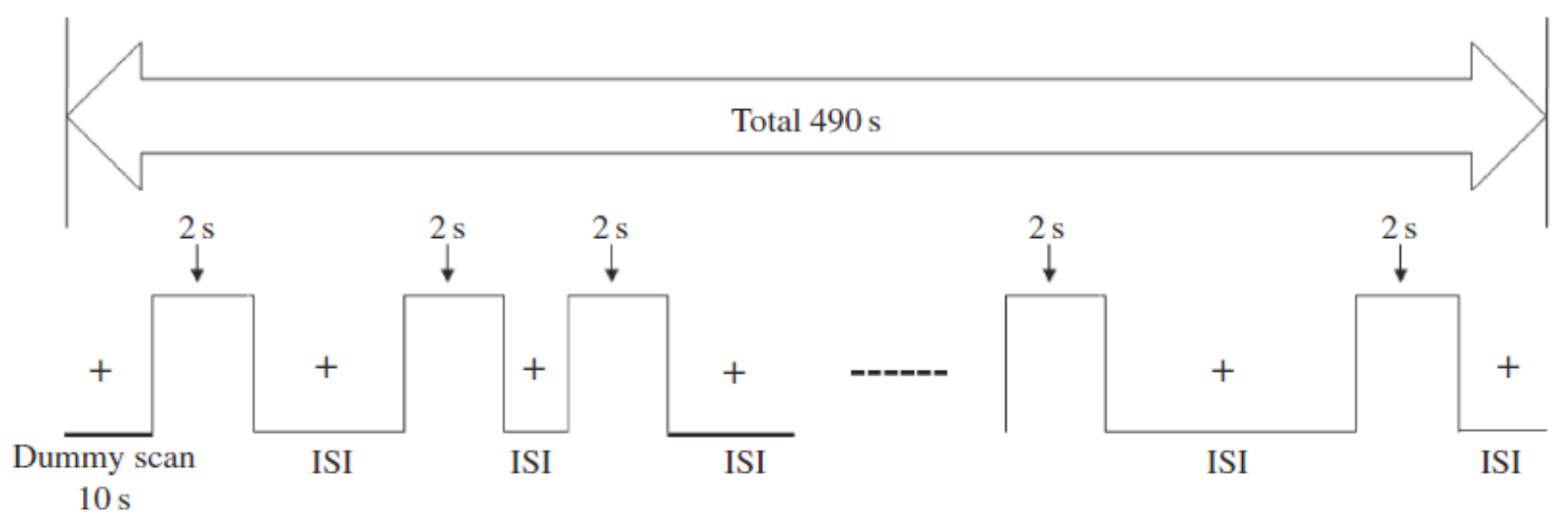

Figure S2 The 2 types of image cues were are presented in pseudorandom order, with each image presented for 2 seconds, followed by an inter-stimulus interval (ISI) ranging from 4 to 12 seconds (mean 8 seconds), during which a white crosshair with black background is presented while magnetic resonance imaging scanning is underway. The total experiment time lasted for 490 seconds. 